\title{
Quality assurance in the emergency room
}

\author{
Frank Oberklaid, Peter Barnett, Frederick Jarman, Jill Sewell
}

Quality assurance programmes have become an accepted part of health care delivery in hospitals in a number of countries. In addition to their value as management and clerical systems designed to ensure that clinical care is of an acceptable standard, ${ }^{1}$ they have also become popular among administrators who see the implementations of quality assurance programmes as a measure of the efficient and effective allocation and use of resources. ${ }^{2}$

There are three general approaches to the development of quality assurance progammes ${ }^{3}$ : (a) structural approaches-for example, addressing issues such as medical education, practitioner training and registration, equipment and facilities, (b) evaluation of the outcomes of health delivery, and (c) documenting and assessing the process of delivering health care.

Most studies of quality assurance in clinical medicine have focused particularly on process and outcome as an index of the quality of care. ${ }^{4}$ For example many existing programmes study such parameters as the adequacy of medical records, patient satisfaction, laboratory utilisation, waiting times, and outcome variables such as mortality, postoperative infection or complication rates, duration and severity of symptoms, and so on. ${ }^{5}$ There have been criticisms of both these approaches. Outcomes are not always easy to measure, and especially in the case of paediatric practice outcomes are not always attributable directly to the care provided ${ }^{6} 7$ : for example children with viral upper respiratory infections are likely to get better irrespective of what type of care they receive. An evaluation of the process of care is usually a judgment rather than a precise objective measurement. ${ }^{8}$ Furthermore, interventions designed to improve the process of health care may not always be congruent with the priority outcome goal of improved health status. For example though it is desirable to decrease patient waiting times, admission rates, and the number of laboratory investigations performed, there comes a point when the pursuit of these process goals may be counterproductive in the context of improving health outcome. Reducing the waiting time should not be at the expense of an unhurried, thorough evaluation of each patient. Nevertheless, despite these shortcomings these approaches generally do provide a means of holding health care providers accountable for following procedures that are, to the best of current knowledge, appropriate and optimal.
The maintenance of high standards of clinical care in paediatric emergency rooms poses particular challenges. These include:

- The high patient attendances, so that doctors and nurses frequently work under considerable pressure to see patients quickly

- The uneven nature of the workload

- The tendency for the emergency room to be staffed by junior staff with relatively little experience

- The rotation of doctors through the emergency room for limited periods of time, and the need for rosters covering the whole 24 hour period, thereby limiting their exposure to and reducing the potential effectiveness of educational activities

- The life threatening nature of many of the presenting conditions.

It is not surprising that quality of care questions are perceived as the least of the problems facing emergency room directors and administrators, and may explain the relative paucity of articles and studies focusing on quality assurance in this setting. ${ }^{7-14}$ However, one could argue that it is because of the difficulties outlined above that emergency quality assurance measures are especially important.

In this paper we describe a programme of such measures developed for the emergency room of a large paediatric teaching hospital. We focus in particular on the structural and process areas of quality assurance and argue that while individually simple and inexpensive to introduce, together they provide a framework for facilitating the delivery of quality clinical care in a paediatric emergency setting.

\section{Background}

The Royal Children's Hospital, Melbourne, is a large, freestanding paediatric teaching hospital which provides the full range of primary, secondary, and tertiary services. Over 70000 children per year are seen in the emergency roomabout $40 \%$ of these are triaged to an adjacent walk in clinic within the department (general clinic). At any one time, 16-18 doctors in their second and third year postgraduation work varying rosters to cover the department on a 24 hour basis. Approximately 60 residents rotate through the department each year. The majority of these are not paediatric trainees but rotate from other hospitals and family practice training programmes for three months of paediatric 
experience. When they arrive in the department the vast majority have had no prior paediatric experience.

In addition a number of registrars (doctors who are in their second to sixth year of paediatric training) are rostered to emergency to provide supervision for the residents and assess children for admission; there are two registrars from 0900-2300 hours and one registrar during the night. The residents work a variable roster with approximately $60 \%$ of their time spent in emergency and the remainder in the general clinic, to which children with less acute/severe conditions are triaged. From 0900-2300, when the general clinic is operational, only children with acute/severe conditions, babies aged less than 1 month, or those presenting because of trauma, are seen in the emergency room. Outside these hours all patients are seen in the emergency room. Because of the range of services provided by the hospital many children present with manifestations of serious tertiary diseases such as congenital heart disease, cystic fibrosis, or metabolic disease. Just over $10 \%$ of all patients seen in the emergency room are admitted, with a further $18 \%$ reviewed either by telephone or in person by departmental staff. The source of referral of all patients is seen in the figure. Four consultant paediatricians work in the department, and although each has extensive responsibilities in other areas, one is available on an on call basis for the emergency room.

This emergency room thus encompasses many of the quality assurance challenges previously mentioned: a large volume of patients many of whom are very sick, inexperienced doctors who change every three months, and variable rosters which make the planning of educational activities difficult.

\section{Conceptual framework}

Several concepts permeated the evolution of various programmes within the department and are reflected in some of the quality assurance measures. Firstly was the concept that the quality of care delivered in the emergency room had to reflect the standards of the hospital as a whole and its individual departments. For example the competence with which children with fractures and other orthopaedic problems were managed in the emergency room should be the equal of the way they were managed in the orthopaedic department. This meant negotiating with individual departments around the hospital for

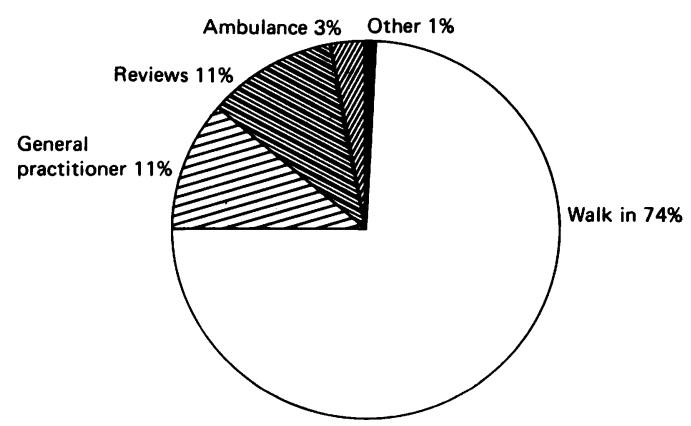

Source of referral. them to share responsiblity for the delivery of care in the emergency room by being involved on a equal basis in the development of clinical protocols and providing a 24 hour on call consultation service to the emergency room.

Secondly a conscious decision was taken that this would not be a 'freestanding' emergency room which operated in a quasi-independent fashion from the rest of the hospital. It was felt that it was unlikely that emergency room staff would ever develop the expertise of, for example, a surgeon or an intensivist. Rather than 'work up' the patient until a diagnosis was firmly established, and initiate investigation and management procedures, it was considered more efficient to request specialty registrars and consultants to be involved in the assessment of these patients in the emergency room at an early stage. If a child with multiple trauma arrived in the emergency room personnel from the inpatient services would be involved with the patients as soon as possible after arrival. This meant that each hospital department gave a clear message to registrars and consultants that consultation to the emergency room was a high priority.

Thirdly the department had a commitment to the health of the children of the whole community, not just those who sought care at the hospital. For example written and oral communication with family doctors was considered important, and this added to the time the doctor spent with each patient. Furthermore the obvious advantage in employing only those residents committed to a full year in paediatric training had to be balanced by our desire to offer paediatric experience to as many family practice trainees as possible.

This conceptual framework guided and influenced the development of the quality assurance programme in the emergency room.

\section{Components of the programme}

The individual components of the quality assurance programme are outlined in table 1 and will be detailed below.

\section{ORIENTATION/EDUCATION}

It is clearly impossible for doctors to deliver quality care if they are unfamiliar with institutional and departmental policies and procedures or if they have an insecure knowledge basis in paediatrics. A great deal of effort is put into orientation and education.

\section{Orientation}

Detailed written orientation guidelines are sent to each resident before commencement-this covers rosters, organisational structures, logistics, teaching and supervision, and other details of their service in the department. On the morning of commencement a two hour long group orientation is conducted by department staff, followed by individual orientations to the emergency room and general clinic. During the subsequent two weeks there are informal personal follow up meetings with each resident to 
Table 1 Individual components of the quality assurance programme

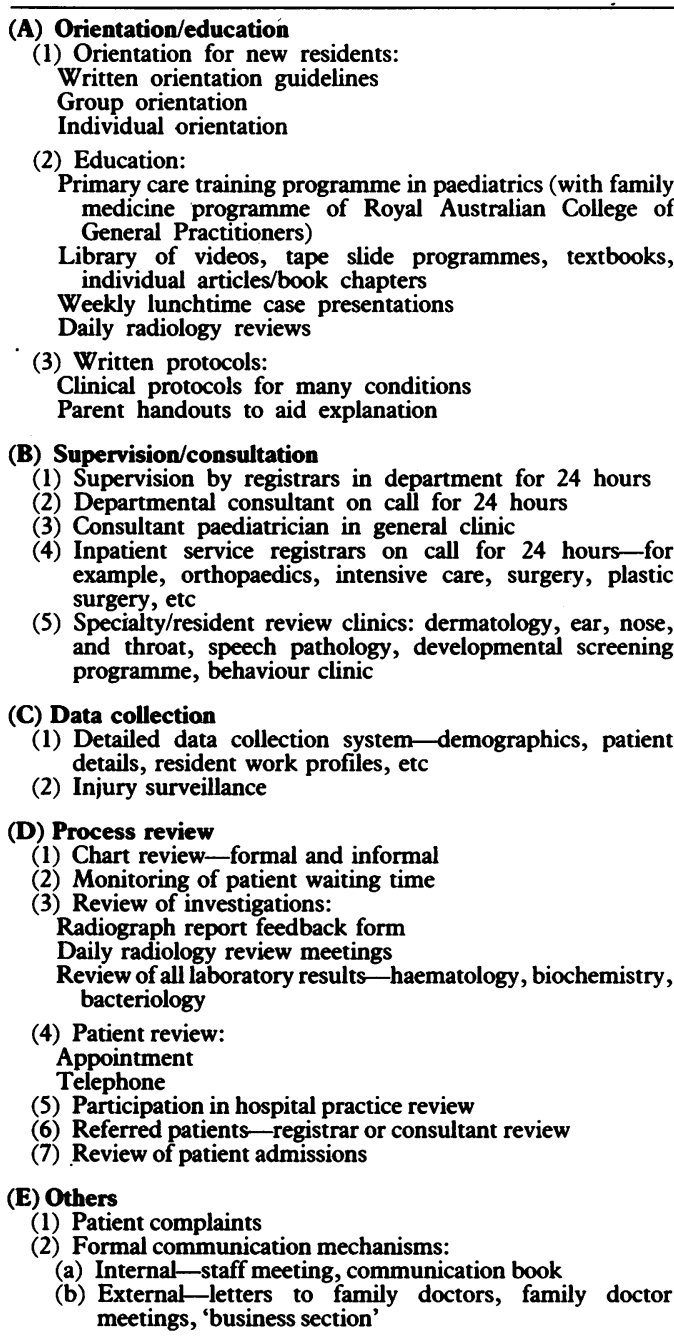

ensure familiarity and comfort within the department.

\section{Education}

In addition to the informal teaching that is part and parcel of most health care systems a number of more structured activities have been organised. A formal curriculum in primary care paediatrics was developed some years ago in conjunction with the family medicine programme of the Royal Australian College of General Practitioners. It consists of 57 one hour tutorials which cover in theory what a practising family doctor needs to know to be reasonably competent in paediatrics; teaching sessions are held each afternoon. Weekly lunchtime case presentations take place, run by one of the consultants, in which residents present and discuss interesting cases seen in the previous week.

Each weekday a radiologist reviews with the group of residents radiographs taken the previous day. An extensive library of textbooks, videos, tape slide programmes, and selected articles and book chapters is kept in the department and used extensively by residents.

Written protocols

Clinical protocols have been developed for approximately 50 conditions and are utilised as departmental guidelines for the management of these conditions. As previously mentioned, most protocols are developed as a joint effort between staff of the department and from the relevant specialty department, for example, gastroenterology and orthopaedics.

To ensure some consistency in the information presented to parents, parent handouts have been developed for many common paediatric conditions (currently 15). They are written at an 11 year old reading level on brightly coloured paper and are given to parents to taken home after their emergency room attendance. These are being distributed to health professionals throughout Australia.

\section{SUPERVISION/CONSULTATION}

Because most doctors working in emergency rooms are relatively junior and inexperienced, supervision and the availability of around the clock consultation are mandatory. Registrar supervision is provided in the emergency room on a continual basis and a departmental consultant is also available. The consultant is on site during normal working hours and then on call after hours and on weekends. In this sense our department probably differs little from others. However, there are three other levels of supervision and consultation which are innovative.

\section{(1) Inpatient service registrars}

As previously mentioned registrars and consultants from the various inpatient services provide a continuous on call service to the emergency room. Most children with fractures, for example, are reviewed by an orthopaedic registrar before any decision about definitive management. A child with epiglottitis or severe croup, or other life threatening illness, is seen immediately by a registrar or consultant from the intensive care unit. Surgical registrars consult with emergency staff for children with abdominal pain, soft tissue trauma, and so on. There are several benefits of this system: the child receives more expert care and.decisions made about diagnosis and treatment are more likely to be appropriate. Unnecessary admissions to hospital are more likely to be avoided and the process of consultation provides important learning experiences for staff of the emergency room.

\section{(2) Consultant paediatrician in general clinic} This has been described previously. ${ }^{15}$ For a number of years consultant paediatricians have been transferred from the outpatient department to the general clinic where they teach and supervise residents. Definitive expertise is provided at the point of first contact for the patient. Many children who would have been referred to outpatients are now effectively managed in the general clinic, usually at the initial visit, and returned for ongoing care to their family doctor. The implications for teaching, role modelling, and quality assurance of having an experienced paediatrician working alongside resident doctors are obvious. After hours this service is pro- 
vided by senior paediatric fellows working in the general clinic. Although these fellows see patients independently, they also are available for consultation with residents.

\section{(3) Specialty/resident reviews clinics}

The principle espoused above of providing definitive consultation in the walk-in clinic rather than an isolated outpatient department was extended to a number of specialties in addition to general paediatrics. Specialist consultant clinics are held regularly for dermatology, ear, nose, and throat, speech pathology, developmental screening, and behaviour problems. Rather than referring a child to the outpatient department the resident arranges for the child to return on one of the specialty clinic days where the designated specialist is available in the general clinic to consult with them. The advantages for teaching, patient and doctor convenience, hospital efficiency, and quality assurance are clear and it is planned to extend this system further.

\section{DATA COLLECTION}

The collection of data is an essential prerequisite to programme evaluation as without the provision of a continuous and reliable data set the provision of efficient services of a high quality is made very difficult. In addition to the usual demographic data (age of child, time of arrival, diagnosis, disposition) information is also collected about consultation, investigations, and complexity of the illness, and data can be analysed individually for all residents (see table 2). We are thus able to build up a 'practice profile' for each doctor. This profile includes how many patients are seen, their diagnoses, how often a consultation with a senior doctor is obtained, how many laboratory investigations are performed and in which context, what proportion of patients are reviewed or referred to outpatients, and so on. This is a valuable teaching aid in that we are able to describe a resident's paediatric experience and identify possible gaps in training. Its use as a quality assurance measure is even more valuable because we can document 'outliers' from average practice. For example if we find a resident bringing back patients for review twice as frequently as his/her colleagues or performing an unusually high proportion of laboratory

Table 2 Prospective data collection in the emergency room

Patient identification numbe

Date of birth

Date

Residential postal code

Source of referral (for example, walk in, family doctor, etc)

Time of arrival

Time of arrival

Resident identification number

Resident identification number

'Siagnosis (abbreviated

'Severity' of disorder

Consultation (for example, emergency room registrar, consultant)
Laboratory investigations (for example, haematology, biochemistry, blood/urine/cerebrospinal fluid cultures, etc) chemistry, blood/urine/cerebrospinal fluid cultures, etc)
Procedures (for example, venepuncture, lumbar puncture, etc) Procedures (for example, venepunct
Time of discharge from department

Disposition (for example, home, no follow up, patient review phone review, review by local doctor, outpatient referral, etc)

${ }^{*}$ ICD-9, International Classification of Diseases, 9th revision. investigations, this will point to the need for that resident's practice to be reviewed as it may be an indication of lack of expertise or confidence. Other similar analyses can be performed in the interests of delivery of high quality paediatric care.

\section{PROCESS REVIEW}

A series of reviews of the process of delivery of care takes place in a number of areas. There are formal and informal chart reviews-each morning the consultant conducts a chart review session with the resident and registrar who have worked the night roster. Informal ad hoc chart reviews are conducted by registrars and consultants on a continual basis.

Similarly, laboratory investigations are reviewed on a formal basis. In addition to daily radiology review meetings residents are given rapid feedback about their interpretation of radiographs by use of the 'radiograph report feedback form' previously described. ${ }^{16}$ These forms are initially returned to the paediatrician responsible for emergency so that he can act on any radiographs that have been interpreted incorrectly by the resident. Sometimes the misinterpretation is of little consequence but at other times it has significant implications for patient management. All laboratory investigations are entered into a central log book and results reviewed formally each day by a 'designated resident'. It is his/her responsibilty to telephone parents with results and change or initiate appropriate treatment. This provides a 'safety net' process that ensures that significant laboratory results (particularly microbiology cultures) are always acted upon.

All patient reviews by residents are centralised in a single appointment book so that they can be monitored. Patient waiting time is monitored and times that exceed predetermined levels are acted upon by the consultant. All patients referred by practitioners in the community are formally reviewed by a consultant or registrar before decisions about disposition, and a consultant sees each chid who is being considered for admission. Finally patients seen in the emergency room are included in those discussed at the formal hospital practice review meetings. ${ }^{17}$

\section{OTHERS}

There are several other items which are included in the department's quality assurance programme. Patient complaints are acted upon formally, documented, and procedures reviewed in an attempt to address any underlying problems that led to the reason for the complaint. Complaints are often used as the basis for weekly resident case discussions. Finally, communication both within the department and with the community is taken seriously. Written and telephone contact with family doctors are encouraged, and a summary letter form has been introduced to facilitate this. At regular bimonthly educational meetings of family doctors at the hospital, a 15 minute 'business section' is conducted where community doctors provide feed- 
back to us about patient care, follow up, discharge letters, and a host of other issues.

Within the department, apart from regular formal staff meetings, there are communication books where staff include information about incidents and problems; these are acted upon the next day by senior staff.

\section{Discussion}

It has been argued that quality assurance is a process, never an event. ${ }^{18} \mathrm{We}$ have attempted to develop quality assurance measures as an underlying philosophy of delivering health care. Given the realities that face us and many other emergency rooms-rotating, inexperienced residents dealing with large numbers of often very ill patients - it was essential for us to provide a 'safety net' of the described measure in an attempt to facilitate quality services.

Some may argue that many common, acute ambulatory problems will resolve themselves satisfactorily whether or not there has been any medical intervention and whether or not that intervention has been appropriate. This might be one of the reasons there are so few reports of quality assurance measures in emergency rooms or in other ambulatory settings. ${ }^{17}$ Indeed most attention has been focused traditionally on inpatient services. In some instances these have been focused on outcome (for example, surgical complications, deaths) but more often it is the process of care examined. In many conditions it is difficult to focus on quality of outcome because of difficulties in accurately measuring changes in a patient's condition, let alone in attributing any changes to the specific intervention. ${ }^{1}$

Our programmes focus particularly on the structure and process of delivering care. Given 16 new residents every three months, as well as a high turnover of rotating registrars, we try to ensure from the outset that quality of clinical care is not compromised by a resident's unfamiliarity with the policies, procedures, and logistics of a new department. The orientation programme is time consuming for everybody involved and is compulsory for all residents, irrespective of their particular shift. Formal evaluations at the completion of resident terms indicate that the orientation has been found to be very valuable. Similarly, the availability of specific clinical protocols provides a structure from the outset for the delivery of consistent, high quality clinical care.

We believe that the level and range of consultation and supervision greatly enhances both teaching and patient care. Ambulatory care at the hospital has been rethought to address the principle of specialist consultant care to be provided to the patient at the point of first contact with the hospital wherever possible. Previously residents would refer children to an outpatient clinic, often weeks or even months away, and meanwhile be uncertain about diagnosis and management. Paediatricians are now available during normal working hours to provide immediate consultation so that a much higher level of expertise is provided. This greatly enhances teaching and significantly improves ihe quality of care. This aspect of the depart- ment consistently receives the highest ratings in resident evaluations of their term in ambulatory paediatrics. It has been suggested that scheduling consultants to work in walk-in clinics in preference to the outpatient department is a far more efficient way to organise services at a hospital level, especially as the majority of referrals to outpatients come from walk-in patients rather than from the community. ${ }^{15}$ The contribution of such an arrangement to quality assurance is a valuable additional benefit.

Although long waiting times are an often accepted feature of hospital emergency rooms because of the imbalance between staff and patient numbers, ${ }^{19}$ and the uneven and unpredictable attendances, it does not necessarily follow that the amount of time residents spend with patients has a major influence on the quality of care received. The availability of appropriate and immediate supervision will greatly facilitate productivity because residents spend less time with assessments, perform fewer unnecessary investigations, and feel more decisive about management. However of great importance also is a data collection system that allows us to develop profiles of resident work. This allows the early identification of those who require more guidance or counselling. With over 60 residents per year it is inevitable there will be a wide range of competence and confidence. The early flagging of those who work differently from their peers allows appropriate interventions to take place and is a major component of our quality assurance measures.

A number of the process reviews that have been implemented are innovative, such as the radiograph report feedback form. ${ }^{16}$ It is in this area that we continue to evolve mechanisms for monitoring the quality of our clinical care. It could be argued that, with some exceptions, ${ }^{15} 17$ the components of these quality assurance mechanisms are qualitative. There are few data to validate their effectiveness. The more difficult next step, notwithstanding the problems previously described, is to evaluate these measures in a quantitative manner against specified outcome criteria. However in an area where reported quality assurance measures are sparse, we have developed a comprehensive series of strategies and processes which greatly increases the likelihood of our providing consistent quality care to children and their families.

Not all components of the quality assurance programme described in this paper are relevant to all emergency rooms. In many hospitals there may be financial, logistic, or political constraints which preclude the introduction of such measures even if they are seen as relevant and desirable. Paediatricians who have involvement with the emergency rooms of general district or community hospitals, for example, may have difficulty providing as much senior level supervision of junior staff as they may wish, or in implementing comprehensive teaching programmes. Nevertheless, the broad concepts of quality assurance are relevant to all and certain key components of our quality assurance programme can probably be applied in most settings.

Accurate, prospective collection of data is 
clearly critical to any quality assurance undertaking. Unless emergency rooms have reliable data on patient numbers and characteristics, the types of medical conditions which present, and the way these conditions are dealt with, many aspects of the department's functioning will lack direction. Standard written protocols which provide a uniformity of approach to common clinical problems are even more important when direct supervision/consultation is not available. Formal staff orientation procedures and access to educational materials are also measures within the capability of most small hospitals.

Paediatricians face the challenge of selecting the quality assurance options most appropriate to the needs of their own hospitals and the population of children and families they serve.

1 Wilson CRM. The four essential components of quality assurance. Hospital-wide quality assurance. Toronto: WB Saunders, 1987:40-51.

2 Australian Council on Hospital Standards. Quality assurance program implementation. Sydney: Australian Council on Hospital Standards, April 1988:5-6.

3 Haggerty RJ, foreward to Cook CD, Heidt J. Assuring quality outpatient care for children. New York: Oxford University Press, 1988.
4 Donabedian A. The quality of medical care. Science 1978; 200:856-63.

5 Howanitz PJ, Howanitz JH. General aspects of laboratory quality assurance. Laboratory quality assurance. New quality assurance. Laboratory quality assurance.

6 Cook CD, Heidt J. Assuring quality outpatient care for children. New York: Oxford University Press, 1988.

$\tau$ Waskerwitz S, Unfer SM. Quality assurance in emergency pediatrics. Pediatr Emerg Care 1987;3:121-6.

8 Brook RH, Stevenson RL Jr. Effectiveness of patient care in Brook RH, Stevenson RL Jr. Effectiveness of patient care

9 Levy R, Goldstein B, Trott A. Approach to quality assurance in an emergency department: a one-year review. Ann Emerg Med 1984;12:166-9.

10 Flint LS, Hammett WH, Martens K. Quality assurance in the emergency department. Ann Emerg Med 1985;14: $134-8$.

11 Harchelroad FP Jr, Martin ML, Kremen RM, et al. Emergency department daily record review: a quality assurance. $Q R B$ 1988;14:45-9.

12 American College of Surgeons Committee on Trauma. Quality assurance and education in the emergency department. Appendix F to hospital resources document. Bulletin of the American College of Surgeons 1980;65:34-5.

13 Sollars GM. Emergency department quality assurance: perspectives from the teaching hospital. Topics in Health Record Management 1984;5:47-53.

14 Anderson GV, Roy A, Looney GL, et al. A unique approach to evaluation of emergency care. FACEP 1977;6:254-8.

15 Oberklaid $F$. Outpatient paediatrics-the need for change. Aust Paediatr $\mathcal{f} 1982 ; 18: 147-8$.

16 Nolan TM, Oberklaid F, Boldt D. Radiological services in a hospital emergency department-an evaluation of service delivery and radiograph interpretations. Aust Paediatr $\mathfrak{I}$ 1984;20:109-12.

17 Smith A, Nolan T. Evaluation of a children's hospital medical peer review programme. Aust Paediatr $\mathcal{F}$ 1989;25:192-5.

18 Greenspan J. Accountability and quality assurance in health care. Bowie, Maryland: Charles Press Publishers, 1980:vii.

19 Smeltzer CH, Curtis L. Analyzing patient time in the emergency department. $Q R B$ 1986;12:380-2. 